\title{
Theory and simulation of nonrelativistic elliptic-beam formation with one-dimensional Child-Langmuir flow characteristics
}

\author{
Ronak Bhatt and Chiping Chen \\ Plasma Science and Fusion Center, Massachusetts Institute of Technology, Cambridge, Massachusetts 02139, USA
}

(Received 2 July 2004; published 3 January 2005)

\begin{abstract}
A theory of nonrelativistic, laminar, elliptic-beam formation is presented. The beam is shown to have the one-dimensional Child-Langmuir flow form. An analytic expression for the electrostatic potential outside the beam is derived. Equipotentials corresponding to electrode surfaces are computed numerically. The effectiveness of the electric field formed by the electrodes in focusing and preserving the elliptic, 1D Child-Langmuir flow beam is verified via 3D ray-tracing simulations.
\end{abstract}

DOI: 10.1103/PhysRevSTAB.8.014201

PACS numbers: 41.75.-i, 41.85.Ar, 52.59.Sa

\section{INTRODUCTION}

Electron beams of elongated elliptic cross sections have generated great interest in vacuum electronics [1] because of their low space-charge energy and efficient coupling to rf structures when compared to circular beams. It is well known that high space charge reduces conversion efficiency in conventional microwave tubes employing circular beams. Presently, there are vigorous activities in the development of sheet-beam traveling wave amplifiers [2,3], klystrons [4], and focusing systems [5,6].

In high-intensity ion and electron accelerators, beams often exhibit nonlaminar flows such as large-amplitude density fluctuations [7], mismatched envelope oscillations [8], emittance growth, chaotic particle orbits, beam interception, and difficulty in beam focusing and compression. Many of these effects are due to beam mismatch or nonequilibrium behavior. Elliptic beams may allow simplified and more natural matching [9] between beam injectors and commonly used magnetic focusing lattices, reducing the emittance growth associated with beam mismatch.

Although elliptic beams present numerous advantages, their inherent three-dimensional nature has made diode design a challenging process, both analytically and numerically. For the applications discussed above, desirable beam characteristics include uniform current density, parallel flow, and zero magnetic flux threading the emitterproperties consistent with one-dimensional ChildLangmuir (C-L) [10] flow, in which the electrostatic potential varies as $\Phi \propto z^{4 / 3}$, where $z$ is the beam propagation distance. In general, however, such flows are difficult to produce [11]. Recent studies of 2D and 3D [12-16] extensions of the Child-Langmuir law in an infinite applied magnetic field have shown that the beam exhibits significant current density enhancements near the beam-vacuum boundary. In the absence of an infinite confining magnetic field, the beam will tend to spread in phase space, resulting in a degradation of beam quality. As we will show in this paper, it is possible to induce the space-charge flow in a 3D system to take the 1D Child-Langmuir flow form by con- structing a 3D external electrode geometry that is consistent with the 1D Child-Langmuir electric field within the beam.

In order to enforce 1D Child-Langmuir [10] flow in a beam interior, the diode design problem requires solving Laplace's equation for the exterior electric potential with Cauchy boundary conditions on the beam-vacuum boundary. General solutions of such elliptic-equation Cauchy problems are difficult or impossible to obtain [11,17], and standard numerical methods fail due to the exponential growth of errors, which is characteristic of such problems. Nonetheless, Pierce [18], in a classic result, analytically solved the exterior problem for an infinite 2D sheet-beam geometry (i.e., neglecting end effects). By employing the Cauchy-Riemann conditions, Pierce could write the potential outside the beam as the real part of a complex analytic function. He found that the function $\Phi \propto \operatorname{Re}\left[(z+i x)^{4 / 3}\right]$, where $x$ is the transverse distance from the beam edge, satisfies the Child-Langmuir condition, $\Phi \propto z^{4 / 3}$, on the beam edge $(x=0)$. This solution possesses an equipotential surface that corresponds to an external focusing electrode - the "Pierce electrode" - which is inclined at a $67.5^{\circ}$ angle ( $\left.3 \pi / 8 \mathrm{rad}\right)$ to the beam.

Later, Radley [19] employed a Laplace transform technique to determine the exterior potential for a beam of circular cross section. Nakai [20] attempted to generalize Radley's technique to the 3D elliptic-beam problem, but neglected the full functional dependence of the angular Mathieu functions, and as a result, arrived at a simple, but incorrect expression for the exterior potential.

In this paper we present a new analytic technique, based on a 3D generalization of [19], to determine elliptic-beam shaping electrodes which may be used independently or in conjunction with 3D modeling codes. A sample set of electrodes is computed explicitly for a one microperveance, large aspect ratio (10:1) elliptic-beam diode. The geometry is tested via 3D OMNITRAK [21] simulations to show nearly ideal 1D Child-Langmuir space-chargelimited flow with high laminarity and a constant elliptic cross section. 
For the design of physical beam diodes, designers, guided by analytic results, make use of ray-tracing codes such as the 2D EGUN [22] and 3D OMNITRAK [21], or particle-in-cell codes such as MICHELLE [23] and WARP [24]. These codes can be used as part of an iterative optimization process in order to arrive at an approximate set of external electrodes which support the desired beam cross section. The analytic elliptic-beam results of this paper facilitate the numerical design of nonaxisymmetric diodes, which are complicated by the presence of an extra dimension in the electrode geometry optimization. In addition, the present analytic results provide a useful protocol for benchmarking new 3D simulation tools.

\section{THEORY}

We consider a nonrelativistic charged-particle beam of length $d$ and elliptic cross section with semimajor axis $a$ and semiminor axis $b$, as shown in Fig. 1. The charged particles are emitted from a flat elliptic plate, held at potential $\Phi=0$, in the $z=0$ plane, and collected by another flat elliptic plate, held at potential $\Phi=\Phi_{d}$, in the $z=d$ plane. The cold fluid equations describing the beam interior are

$$
\begin{gathered}
\partial n / \partial t+\nabla \cdot(n \mathbf{V})=0, \\
\partial \mathbf{V} / \partial t+(\mathbf{V} \cdot \nabla) \mathbf{V}=-(q / m) \nabla \Phi, \\
\nabla^{2} \Phi=-4 \pi q n,
\end{gathered}
$$

for the region $x^{2} / a^{2}+y^{2} / b^{2} \leq 1$ and $0 \leq z \leq d$. In Eqs. (1)-(3), $\mathbf{V}$ is the flow velocity, and $n$ is the density of particles, each of mass $m$ and charge $q$. Note that,

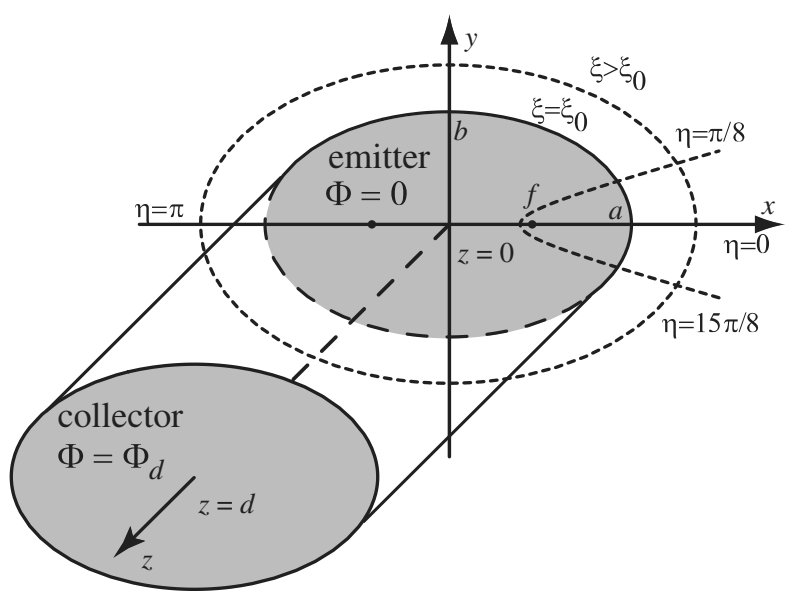

FIG. 1. A beam of elliptic cross section (semimajor axis $a$, semiminor axis $b$ ) is shown in Cartesian and elliptic-cylindrical coordinates. The beam is emitted from an elliptic plate at $\Phi=0$ in the $z=0$ plane and collected by an elliptic plate at $\Phi=\Phi_{d}$ in the $z=d$ plane. The beam fills the area enclosed by the surface $\xi=\xi_{0}$. In any $z$ plane, lines of constant $\xi$ are ellipses, and lines of constant $\eta$ are semihyperbolas. consistent with the nonrelativistic approximation, we neglect any self-magnetic field. In the beam exterior, the potential satisfies Laplace's equation

$$
\nabla^{2} \Phi=0
$$

The 1D steady-state solution to the interior problem defined by Eqs. (1)-(3) can be obtained by using the plate potentials as boundary conditions for Poisson's equation and imposing the constraint that particles emerge from the $\Phi=0$ emitter with zero velocity, i.e., the space-chargelimited boundary condition. This results in the well-known 1D Child-Langmuir [10] solution for laminar, spacecharge-limited flow with $\mathbf{V}(z)=\hat{\mathbf{e}}_{z}\left(2 q \Phi_{d} / m\right)^{1 / 2}(z / d)^{2 / 3}$, $n(z)=\left(\Phi_{d} / 9 \pi q d^{2}\right)(z / d)^{-2 / 3}$, and $\Phi(z)=\Phi_{d}(z / d)^{4 / 3}$. For example, an electron diode of length $d=5.2 \mathrm{~mm}$ and diode voltage $\Phi_{d}=5 \mathrm{kV}$ produces a current density of $3 \mathrm{~A} / \mathrm{cm}^{2}$, while a $\mathrm{Cs}^{+}$ion diode of the same length and voltage generates a current density of $6 \mathrm{~mA} / \mathrm{cm}^{2}$.

To determine the potential distribution in the beam exterior, we solve Laplace's equation (4) while matching the interior and exterior electric fields on the elliptic-beam boundary. From the C-L solution, the matching conditions on the elliptic-beam boundary imply, for $0 \leq z \leq d$,

$$
\left.\Phi\right|_{\text {beam bound }}=\Phi_{d}(z / d)^{4 / 3},
$$

$$
\left.(\hat{\mathbf{n}} \cdot \nabla) \Phi\right|_{\text {beam bound }}=0,
$$

where $\hat{\mathbf{n}}$ is a unit vector normal to the elliptic-beam boundary $x^{2} / a^{2}+y^{2} / b^{2}=1$. We aim to find exterior equipotential surfaces corresponding to the emitter and collector potentials $\Phi=0$ and $\Phi=\Phi_{d}$, respectively. If electrodes at the given potentials are made to lie along these surfaces, they will enforce the conditions in Eqs. (5) and (6) on the interval $0 \leq z \leq d$.

It is useful to introduce the elliptic-cylindrical coordinate $\operatorname{system}(\xi, \eta, z)$, i.e.,

$$
x=f \cosh (\xi) \cos (\eta), \quad y=f \sinh (\xi) \sin (\eta), \quad z=z,
$$

where $0 \leq \xi<\infty$ is a radial coordinate, $0 \leq \eta<2 \pi$ is an angular coordinate, and $f=\sqrt{a^{2}-b^{2}}$ is the distance from the center of the ellipse to either of its foci, as illustrated in Fig. 1. The elliptic-beam boundary is specified by the surface $\xi=\xi_{0}=\operatorname{coth}^{-1}(a / b)$. In these coordinates, Laplace's equation may be expressed

$$
\frac{2}{f^{2}(\cosh 2 \xi-\cos 2 \eta)}\left(\frac{\partial^{2} \Phi}{\partial \eta^{2}}+\frac{\partial^{2} \Phi}{\partial \xi^{2}}\right)+\frac{\partial^{2} \Phi}{\partial z^{2}}=0
$$

and the boundary conditions in Eqs. (5) and (6) are

$$
\left.(\partial \Phi / \partial \xi)\right|_{\xi=\xi_{0}}=0
$$




$$
\begin{gathered}
\left.(\partial \Phi / \partial \eta)\right|_{\xi=\xi_{0}}=0, \\
\left.\Phi\right|_{\xi=\xi_{0}}=\Phi_{d}(z / d)^{4 / 3} .
\end{gathered}
$$

Expressing a product solution as $\Phi(\xi, \eta, z)=$ $\Xi(\xi) \Theta(\eta) \mathrm{Z}(z)$ and applying separation of variables to Eq. (8), we find

$$
\begin{gathered}
d^{2} \mathrm{Z} / d z^{2}-k^{2} \mathrm{Z}=0, \\
d^{2} \Theta / d \eta^{2}+\left(\lambda-\frac{1}{2} k^{2} f^{2} \cos 2 \eta\right) \Theta=0, \\
d^{2} \Xi / d \xi^{2}-\left(\lambda-\frac{1}{2} k^{2} f^{2} \cosh 2 \xi\right) \Xi=0,
\end{gathered}
$$

where $k$ and $\lambda$ are separation constants. Equation (12) leads simply to exponential solutions $\mathrm{Z}=e^{k z}$. Equation (13) is the angular Mathieu equation, but we are only interested in those angular Mathieu functions which have a periodicity of $2 \pi$ and are even about $\eta=0$ and $\eta=\pi / 2$, since the boundary conditions of Eqs. (10) and (11) possess these same symmetries. Such solutions exist only for discrete eigenvalues of the separation constant $\lambda$, and we adopt the convention of Morse and Feshbach [25] to denote these angular Mathieu functions by $\Theta=S e_{2 n}(k f, \eta)$ and the associated normalization constants $M_{2 n}^{e} \equiv$ $\int_{0}^{2 \pi}\left[\operatorname{Se}_{2 n}(k f, u)\right]^{2} d u$, where $n$ is a non-negative integer indexing the eigenvalues $\lambda$, as detailed in Ref. [25]. The corresponding radial solutions, $\Xi=J e_{2 n}(k f, \xi)$ and $\Xi=$ $N e_{2 n}(k f, \xi)$, are radial Mathieu functions of the first and second kind, respectively.

Any superposition of product solutions of the separated equations must satisfy Laplace's equation (8), and hence we write

$$
\Phi(\xi, \eta, z)=\int_{C} d k A(k) e^{k z} G(k f, \xi, \eta),
$$

where

$$
\begin{aligned}
G(h, \xi, \eta) \equiv & \sum_{n=0}^{\infty} \alpha_{2 n}(h) S e_{2 n}(h, \eta)\left[J e_{2 n}(h, \xi) N e_{2 n}^{\prime}\left(h, \xi_{0}\right)\right. \\
& \left.-N e_{2 n}(h, \xi) J e_{2 n}^{\prime}\left(h, \xi_{0}\right)\right] .
\end{aligned}
$$

We have chosen $\alpha_{2 n}(h) \equiv\left[M_{2 n}^{e}\right]^{-1} \int_{0}^{2 \pi} S_{2 n}(h, u) d u$, and the primes denote differentiation with respect to $\xi$. The corresponding expression in Nakai [20] does not have $\operatorname{Se}_{2 n}(h, \eta)$ and omits the normalization factor $\alpha_{2 n}$.

The expansion in Eq. (15) assures that $\Phi$ satisfies Laplace's equation (8), and it is readily seen that the particular linear combination of radial Mathieu functions in Eq. (16) satisfies the boundary condition in Eq. (9). Moreover, using the Wronskian for the radial Mathieu functions and the orthogonality of the angular Mathieu functions, it can be shown that our definition of $\alpha_{2 n}$ assures $G\left(h, \eta, \xi_{0}\right)=1$, and thereby the boundary condition in Eq. (10). Equations (11) and (15) now imply

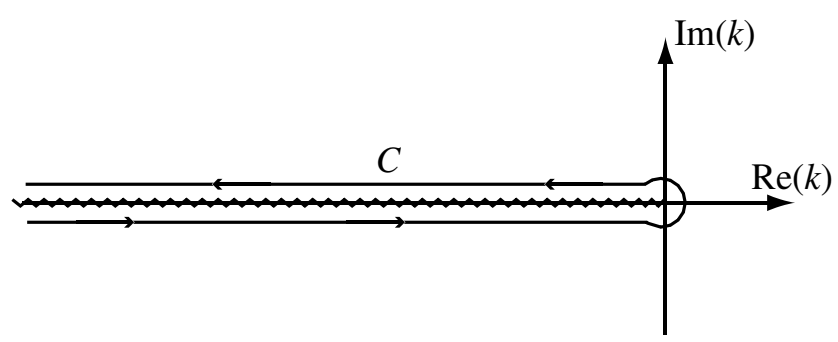

FIG. 2. The Hankel contour in the complex $k$ plane is taken around the $-\infty<k<0$ branch cut.

$$
\Phi\left(\xi_{0}, \eta, z\right)=\int_{C} A(k) e^{k z} d k=\Phi_{d}(z / d)^{4 / 3} .
$$

We follow the analysis by Radley [19] and make use of the integral representation of the gamma function $\Gamma(u)$ [26] to obtain

$$
z^{4 / 3}=\frac{1}{\Gamma\left(-\frac{4}{3}\right)} \frac{i}{2 \sin \left(\frac{4 \pi}{3}\right)} \int_{C} e^{k z} k^{-7 / 3} d k
$$

where the Hankel contour $C$ is taken around the branch cut defined by the line $-\infty<k<0$, as shown in Fig. 2 . Substituting Eq. (18) into Eq. (17), we find that

$$
A(k)=\frac{\Phi_{d} d^{-4 / 3}}{\Gamma\left(-\frac{4}{3}\right)} \frac{i}{2 \sin \left(\frac{4 \pi}{3}\right)} k^{-7 / 3},
$$

provided the same Hankel contour is used for the integration. Having determined $A(k)$, the expression for the exterior potential in Eq. (15) is fully specified.

\section{SIMULATION}

The prescription of Sec. II can be used to compute the potential outside a 1D Child-Langmuir flow beam of arbitrary elliptic-cylindrical geometry. However, for definiteness, we use the above technique to compute the potential outside a 10:1:8.7 space-charge-limited elliptical diode of semimajor axis $a=10 b$, semiminor axis $b$, and length $d=8.7 b$. Equipotentials corresponding to $\Phi=0$ and $\Phi=\Phi_{d}$ are shown in Figs. 3(a) and 3(b), respectively, for several values of $z$. The level contours are roughly elliptical in shape, and the $\Phi=\Phi_{d}$ surface is more steeply inclined to the beam than the $\Phi=0$ surface, as expected from the 2D theory [18]. The equipotentials in Fig. 3 were computed by applying a numeric root-finding scheme to the potential defined in Eq. (15). The Hankel contour integral is numerically evaluated employing standard techniques for the evaluation of the Mathieu functions [17,27]. It should be noted that these results differ significantly from those obtained using the method of Nakai [20]. For example, the $z / b=3.3$ equipotential of Fig. 3(a) intersects the $x$ axis at $x / b=33.2$ and the $y$ axis at $y / b=9.2$. The same $z / b=3.3$ equipotential, computed using Nakai's expression, incorrectly gives an ellipse which intersects the axes at $x / b=15.7$ and $y / b=12.1$, respectively. 

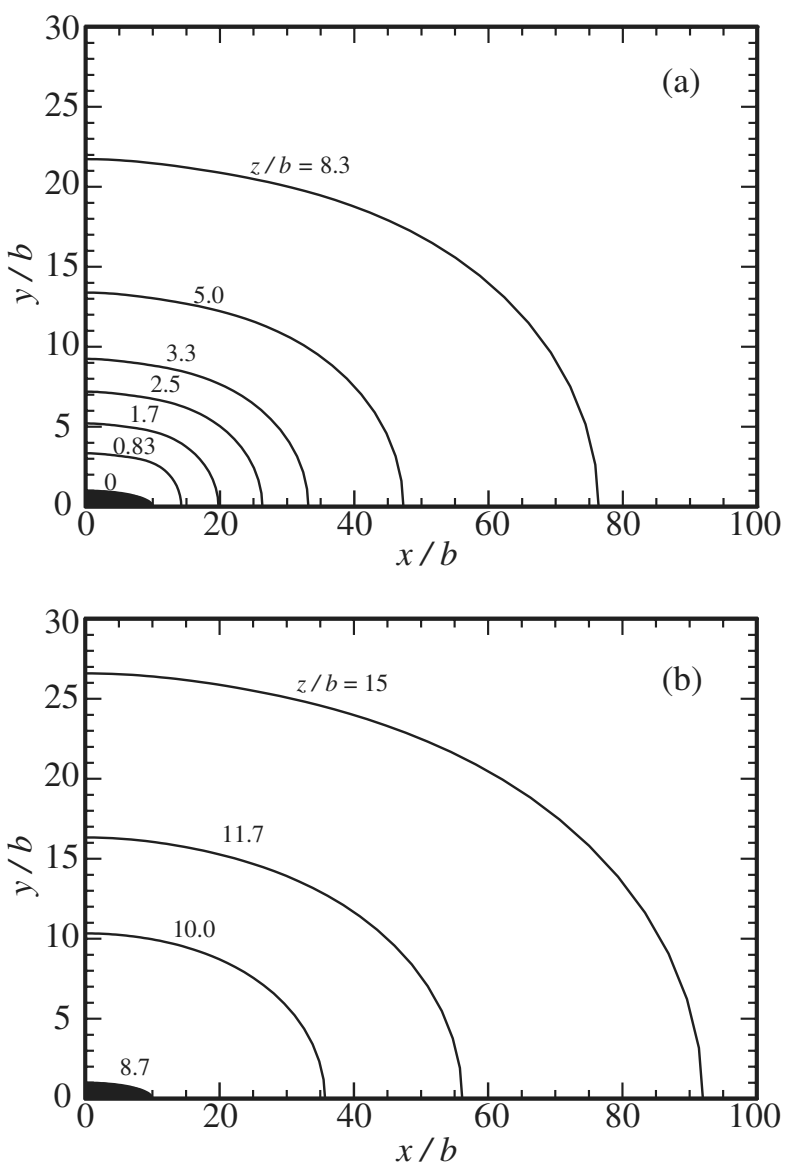

FIG. 3. Level curves of (a) $\Phi=0$ and (b) $\Phi=\Phi_{d}$ surfaces for a 10:1:8.7 space-charge-limited elliptical diode of semimajor radius $10 b$, semiminor radius $b$, and length $8.7 b$. The beam fills the shaded area, which is intersected by the $\Phi=0$ surface at $z=0$ and the $\Phi=\Phi_{d}$ surface at $z=8.7 b$.

We verify our theory by a cold-beam 3D space-chargelimited emission simulation using OMNITRAK [21], a commercially available ray-tracing code. Simulation results are shown in Fig. 4 for the same geometry as in Fig. 3, using a variable-resolution computational mesh with $x$ spacing of $0.1 \mathrm{~mm}$ for $0 \leq x \leq 8 \mathrm{~mm}$ and $0.5 \mathrm{~mm}$ for $8 \leq x \leq$ $15 \mathrm{~mm}, y$ spacing of $0.05 \mathrm{~mm}$ for $0 \leq y \leq 1 \mathrm{~mm}$, $0.1 \mathrm{~mm}$ for $1 \leq y \leq 5 \mathrm{~mm}$, and $0.4 \mathrm{~mm}$ for $5 \leq y \leq$ $12 \mathrm{~mm}$, and $z$ spacing of $0.05 \mathrm{~mm}$ for $0 \leq z \leq 0.8 \mathrm{~mm}$, $0.02 \mathrm{~mm}$ for $0.8 \leq z \leq 1.2 \mathrm{~mm}, 0.05 \mathrm{~mm}$ for $1.2 \leq z \leq$ $2 \mathrm{~mm}, 0.1 \mathrm{~mm}$ for $2 \leq z \leq 5.7 \mathrm{~mm}, 0.05 \mathrm{~mm}$ for $5.7 \leq$ $z \leq 7 \mathrm{~mm}$, and $0.1 \mathrm{~mm}$ for $7 \leq z \leq 10 \mathrm{~mm}$. The mesh resolution is higher in $x$ and $y$ across the cross section of the beam, and in $z$ where the beam intersects the emitter and collector. The 3D electrode structure was linearly interpolated between the equipotentials in Fig. 3, each sampled at 46 points evenly distributed in $\eta$, for $0 \leq \eta \leq$ $\pi / 2$. Nearby computational nodes are shifted to conform to the electrode surfaces using the OMNITRAK surface flag. Neumann boundaries were used for the symmetry planes of the beam as well as for the outer boundaries of the simu-
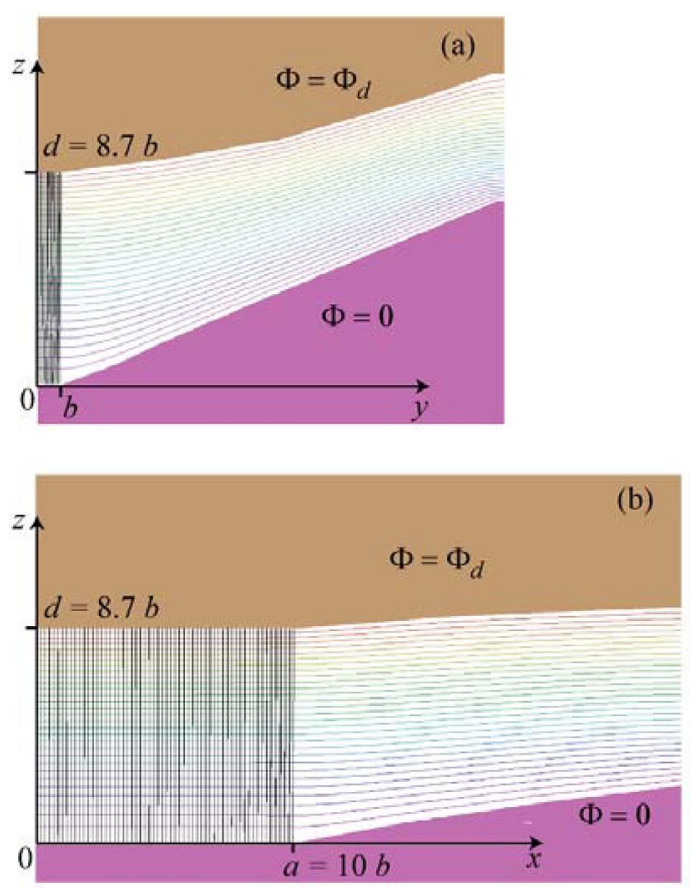

FIG. 4. (Color) A 3D OMNITRAK simulation of space-chargelimited Child-Langmuir flow using the 10:1:8.7 elliptical diode geometry shown in Fig. 3. Particle trajectories and equipotentials are shown in (a) the $x=0$ plane and (b) the $y=0$ plane.

lation region, which is shown in Fig. 4 along with computed equipotentials and particle trajectories projected to the $x=0$ and $y=0$ planes. The entire simulation runs in approximately $30 \mathrm{~min}$ on a $3 \mathrm{GHz}$ personal computer.

The beam produced by the simulation is essentially parallel, laminar, uniform density Child-Langmuir flow. Beam laminarity is often characterized by the rms beam emittances $\varepsilon_{x} \equiv\left(\left\langle x^{2}\right\rangle\left\langle x^{\prime 2}\right\rangle-\left\langle x x^{\prime}\right\rangle^{2}\right)^{1 / 2}$ and $\varepsilon_{y} \equiv\left(\left\langle y^{2}\right\rangle \times\right.$ $\left.\left\langle y^{\prime 2}\right\rangle-\left\langle y y^{\prime}\right\rangle^{2}\right)^{1 / 2}$, where the averages of transverse particle position $(x, y)$ and divergence $\left(x^{\prime}, y^{\prime}\right) \equiv(d x / d z, d y / d z)$ are taken over a slice of the beam at $z=d$. For a uniform density elliptic beam, these emittances can be related to effective beam temperatures [28] by the relations $\varepsilon_{x}=$ $a\left(k T_{\text {eff }, x} / 8 q \Phi_{d}\right)^{1 / 2}$ and $\varepsilon_{y}=b\left(k T_{\text {eff }, y} / 8 q \Phi_{d}\right)^{1 / 2}$. If we use the parameters of our earlier examples, a 10:1:8.7 elliptical electron or ion diode with $\Phi_{d}=5.0 \mathrm{kV}, d=$ $5.2 \mathrm{~mm}, a=6.0 \mathrm{~mm}$, and $b=0.6 \mathrm{~mm}$, the cold-beam OMNITRAK simulation shown in Fig. 4 predicts the effective beam temperatures $T_{\text {eff }, x}=6.7 \times 10^{-4} \mathrm{eV}$ and $T_{\text {eff }, y}=$ $8.1 \times 10^{-3} \mathrm{eV}$. For a realistic beam, of course, the lower limit of the effective beam temperature (and emittance) will be given by the physical temperature of the emitter, approximately $0.1 \mathrm{eV}$ for a typical thermionic electron or ion diode. Since the simulated temperatures are negligibly small compared to this value, we can infer that the emittance of an elliptical diode constructed using the above prescription will approach the theoretical limits imposed by finite emitter temperature. 
For most cases of interest with highly elliptic beams, the focusing electrode slopes are nearly straight lines, except for a small region near the beam edge. Thus, in practice, very usable electrodes for the 10:1:8.7 beam can be designed even when omitting several intermediate equipotentials in the interpolation. For example, for the 10:1:8.7 beam discussed above, we may interpolate directly between the $z / b=0, z / b=0.83$, and $z / b=8.3$ equipotentials of Fig. 3(a) without measurably affecting the simulated emittance. For a somewhat rounder 6:1:6.6 aspect ratio example, we find the emittance increases by approximately $15 \%$ when we omit intermediate equipotentials, which is similar to the results for cylindrical beams.

Our ongoing studies include extending the theory to the relativistic regime. In addition, engineering considerations generally require a thermally insulating gap between the emitter edge and the $\Phi=0$ electrode, which will introduce a perturbation to the beam. Nonetheless, for small gaps, we do not expect a significant modification of the bulk flow. Preliminary simulation studies of the effect of collector hole lensing are encouraging. Although the introduction of a hole through which the beam is extracted alters the nearby field lines, the field perturbation is nearly linear. As a result, the beam divergence is well correlated with position such that emittance growth is modest (factor of 2 or less), which is still negligible.

\section{CONCLUSION}

To summarize, a theory of nonrelativistic, laminar, elliptic-beam formation was presented. The beam was shown to have the one-dimensional Child-Langmuir flow form. An analytic expression for the electrostatic potential outside the beam was derived. Equipotentials corresponding to electrode surfaces were computed numerically. The effectiveness of the electric field formed by the electrodes in focusing and preserving the elliptic, 1D Child-Langmuir flow beam was verified via 3D ray-tracing simulations.

\section{ACKNOWLEDGMENTS}

This work was performed under the auspices of U.S. Department of Energy, Office of High-Energy Physics, Grant No. DE-FG02-95ER40919, Office of Fusion Energy Science, Grant No. DE-FG 02-01-ER54662, Air Force Office of Scientific Research Grant No. F49620-031-0230, and the Deshpande Center for Technological Innovation.
[1] P. A. Sturrock, J. Electron. Control 7, 162 (1959).

[2] S. Humphries, S. Russell, B. Carlsten, and L. Earley, Phys. Rev. ST Accel. Beams 7, 060401 (2004).

[3] B. E. Carlsten, Phys. Plasmas 9, 5088 (2002).

[4] G. Caryotakis et al., in 6th Workshop on High Energy Density and High Power RF, edited by S. H. Gold and G. S. Nusinovich, AIP Conf. Proc. No. 691 (AIP, New York, 2003), p. 22.

[5] M. A. Basten and J.H. Booske, J. Appl. Phys. 85, 6313 (1999).

[6] R. Pakter and C. Chen, Phys. Rev. E 62, 2789 (2000).

[7] S. Bernal et al., Phys. Plasmas 11, 2907 (2004).

[8] T. P. Wangler et al., Nucl. Instrum. Methods Phys. Res., Sect. A 519, 425 (2004).

[9] C. Chen, R. Pakter, and R. C. Davidson, Nucl. Instrum. Methods Phys. Res., Sect. A 464, 518 (2001).

[10] C. D. Child, Phys. Rev. 32, 492 (1911); I. Langmuir, ibid. 21, 419 ( 1923).

[11] P. Kirstein, G. Kino, and W. Waters, Space Charge Flow (McGraw-Hill, New York, 1967), p. 331.

[12] J. W. Luginsland, Y. Y. Lau, R. J. Umstattd, and J. J. Watrous, Phys. Plasmas 9, 2371 (2002).

[13] J. J. Watrous, J.W. Luginsland, and M. Frese, Phys. Plasmas 8, 4202 (2001).

[14] J. W. Luginsland, Y. Y. Lau, and R. M. Gilgenbach, Phys. Rev. Lett. 77, 4668 (1996).

[15] R. J. Umstattd and J. W. Luginsland, Phys. Rev. Lett. 87, 145002 (2001).

[16] Y. Y. Lau, Phys. Rev. Lett. 87, 278301 (2001).

[17] P. Morse and H. Feshbach, Methods of Theoretical Physics (McGraw-Hill, New York, 1953), Vol. 1, Chap. 6, p. 702.

[18] J.R. Pierce, Theory and Design of Electron Beams (Van Nostrand, Princeton, 1954), 2nd ed.

[19] D. E. Radley, J. Electron. Control 4, 125 (1957).

[20] A. Nakai, Nucl. Instrum. Methods 54, 57 (1967).

[21] OMNITRAK software, copyright Field Precision, Albuquerque, NM.

[22] W.B. Hermannsfeldt, SLAC Report No. SLAC 226, 1979.

[23] J. Petillo et al., IEEE Trans. Plasma Sci. 30, 1238 (2002).

[24] D. P. Grote, A. Friedman, I. Haber, W. Fawley, and J. L. Vay, Nucl. Instrum. Methods Phys. Res., Sect. A 415, 428 (1998).

[25] See Ref. [17], Vol. 2, Chap. 11, p. 1568.

[26] See Ref. [17], Vol. 1, Chap. 4, p. 421.

[27] J.C. Gutierrez-Vega et al. (unpublished).

[28] J.D. Lawson, Physics of Charged Particle Beams (Clarendon, Oxford, 1988), p. 210. 